\title{
Tweezing and manipulating micro- and nanoparticles by optical nonlinear endoscopy
}

\author{
Min Gu, Hongchun Bao, Xiaosong Gan, Nicholas Stokes and Jingzhi Wu
}

The precise control and manipulation of micro- and nanoparticles using an optical endoscope are potentially important in biomedical studies, bedside diagnosis and treatment in an aquatic internal organ environment, but they have not yet been achieved. Here, for the first time, we demonstrate optical nonlinear endoscopic tweezers (ONETs) for directly controlling and manipulating aquatic micro- and nanobeads as well as gold nanorods. It is found that two-photon absorption can enhance the trapping force on fluorescent nanobeads by up to four orders of magnitude compared with dielectric nanobeads of the same size. More importantly, two-photon excitation leads to a plasmon-mediated optothermal attracting force on nanorods, which can extend far beyond the focal spot. This new phenomenon facilitates a snowball effect that allows the fast uploading of nanorods to a targeted cell followed by thermal treatment within 1 min. As two-photon absorption allows an operation wavelength at the center of the transmission window of human tissue, our work demonstrates that ONET is potentially an unprecedented tool for precisely specifying the location and dosage of drug particles and for rapidly uploading metallic nanoparticles to individual cancer cells for treatment.

Light: Science \& Applications (2014) 3, e126; doi:10.1038/lsa.2014.7; published online 3 January 2014

Keywords: endoscopy; gold nanorods; micro/nanotweezing; two-photon absorption

\section{INTRODUCTION}

Micro- and nanoparticles, including dielectric and fluorescent beads and metallic nanorods, when they are functionalized, are usually immersed in the fluid of a human body in many biomedical applications. ${ }^{1-8}$ They normally move without control, which reduces their functionality and efficiency, for example, in the treatment of diseases using drug particles ${ }^{4}$ and in diagnosis based on the specificity of fluorescent particles for labeling particular molecules. ${ }^{9}$ Optical tweezing is a useful technique for the non-contact and non-invasive manipulation of small particles in fluid. ${ }^{1-3,10-13}$ However, current optical tweezing instruments are based on a bulky bench-top microscopy system, where a high numerical-aperture (NA) microscope objective is used to generate a tight focus for inducing a large gradient trapping force. ${ }^{10-13}$ Such a bulky objective limits the working distance and prevents optical tweezing from in vivo applications, especially inside an internal organ of a human body.

On the other hand, optical endoscopy, employing a small probe, can enter a human body for in vivo operation ${ }^{14-17}$ and has been successfully used to guide a myriad of diagnostic and therapeutic procedures. In addition, scanning optical endoscopy can precisely locate a focus spot to any position within a large operating volume. ${ }^{6,15}$ However, the low NA of a small probe significantly reduces the gradient force in the focus, which prevents us from efficiently tweezing micro- and nanoparticles. To overcome this major hurdle, we have adopted a low NA optical endoscope ${ }^{15}$ where a near-infrared ultrashort pulsed beam is used to induce two-photon absorption. Two-photon absorption allows the use of illumination wavelengths at the center of the transmission window of human tissues, greatly reducing the phototoxicity, which is one of the most challenging problems in photothermal therapy. We show that tweezing and manipulating micro- and nanobeads and nanorods can be achieved using two-photon excitation. In particular, the localization induced by the two-photon absorption of fluorescent nanobeads and nanorods can enhance the trapping force by 3-4 orders of magnitude. Importantly, for tweezing nanorods, a snowball effect can occur, in which a large number of nanorods distant from the focal spot can be pushed toward the focus due to the strong absorption caused by the surface plasmon resonance of the nanorods. Our numerical analysis reveals that the physical mechanism for the observed snowball effect should be the optothermal attracting force originating from the dynamic increase in the environmental temperature around the trapped nanorods within the focus. Finally, we apply the snowball effect to thermally treat a targeted cell dispersed together with nanorods in water.

\section{METHODS AND MATERIALS}

The experimental demonstration for tweezing and manipulating micro- and nanobeads is carried out in a fiber-optical nonlinear endoscope comprising a microprobe, a double-clad fiber and a control unit connected to a computer. ${ }^{15}$ To observe the tweezing performance, we use a He-Ne laser and a CCD camera. The trapping efficiency is calculated by measuring the maximum velocity at which a trapped particle escapes using the equations provided in the Supplementary Information (Equations (1) and (2)). To reveal the mechanism of the snowball effect, we perform a numerical simulation on the temperature 
variation around the focal spot in which the nanorods are trapped. The simulation is based on the finite-difference time-domain (FDTD) software from Lumerical to calculate the electric field enhancement surrounding a gold nanorod at its longitudinal resonance wavelength. The FDTD simulation is generalized to include the thermal conversion and the conduction process in water. This physical process is modeled by the Green's function approach (see Supplementary Information).

To demonstrate the trapping capability of a nonlinear optical endoscope, we perform optical tweezing by placing an optical nonlinear endoscopic probe into water in which micro- and nanobeads or gold nanorods are immersed (Figure 1). The details of the probe can be found elsewhere $e^{6,15}$ or in the Supplementary Information. Figure 1 shows the experimental set-up of optical nonlinear endoscopic tweezer (ONET), including a microprobe, a length of double-clad fiber and a control unit connected to a computer. ${ }^{6,15}$ For the observation of the trapping performance, the light from another light beam (a He-Ne laser) is scattered by the tweezed particles immersed in water and visualized by a CCD camera through magnification by a $40 \times /$ $\mathrm{NA}=0.6$ dry objective lens. A bandpass filter (FF01-750 SP-25; Semrock, Inc. 3625 Buffalo Road, Suite 6 Rochester, NY 14624) is employed to block the pulsed beam of the nonlinear optical endoscope from reaching the CCD camera. The dielectric beads are from Polybead (Polysciences, Inc., 400 Valley Road Warrington, PA 18976), and the fluorescent beads are from Fluoresbrite Yellow Green Microspheres (Polysciences, Inc.). The preparation and the properties of the gold nanorods are detailed in Supplementary Fig. S1.

\section{RESULTS AND DISCUSSION}

First, we demonstrate the trapping capability of a nonlinear optical endoscope at the low NA focusing condition. It is experimentally shown that both dielectric and fluorescent beads of diameters between $100 \mathrm{~nm}$ and $25 \mu \mathrm{m}$ can be trapped and manipulated by ONET

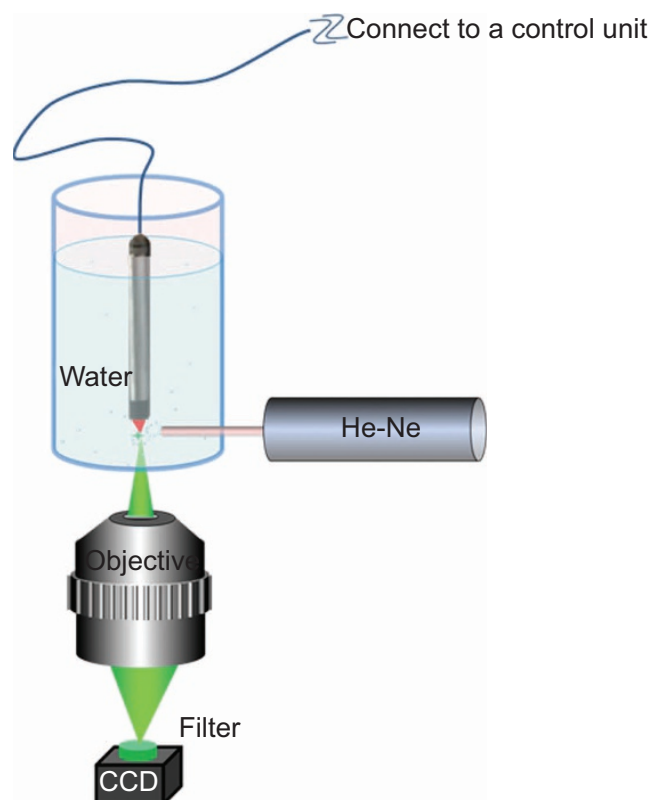

Figure 1 Schematic diagram of ONETs for manipulating and controlling microand nanobeads and gold nanorods. An optical nonlinear endoscopy probe is delivered into a container in which micro- and nanobeads and nanorods are dispersed in water. Tweezing is visualized by a CCD camera and a He-Ne laser. ONET, optical nonlinear endoscopic tweezer.
(Supplementary Figs. S2 and S3). While the control of large fluorescent beads by ONET is demonstrated in Supplementary Fig. S2b1-b4, Figure 2 shows the simultaneous two-photon excitation and tweezing of fluorescent beads of diameters $5 \mu \mathrm{m}$ and $100 \mathrm{~nm}$ by the low NA optical nonlinear endoscope probe. It can be seen that fluorescence is generated when the ultrafast pulsed laser beam hits a fluorescent microbead (Figure 2a2) and that the fluorescing bead is subsequently trapped (Figure 2a3). By moving trapped fluorescent beads, we can arrange them into a letter 'B' (Figure 2a4 and Supplementary Movie 1). The dependence of the fluorescence intensity on the trapping power on a logarithmic scale fits a straight line (Supplementary Fig. S1b) with a gradient of 1.97 , showing that the fluorescence from the bead is generated through two-photon absorption. Similarly, Figure 2b1-2b4 demonstrate that fluorescent nanobeads of diameter $100 \mathrm{~nm}$, trapped by two-photon absorption, can be arranged into a semicircle shape (see Supplementary Movie 2).

It is important to point out that two-photon absorption leads to a pronounced difference in the trapping efficiency's dependence on the diameter between dielectric and fluorescent beads, particularly for nanobeads (Figure 2c). In the case of dielectric beads, the trapping efficiency under the low NA illumination condition decreases when their size is reduced, which is consistent with the previous observation for high NA objective trapping. ${ }^{18}$ Because the NA of nonlinear optical endoscopy is low, the trapping efficiency of dielectric beads is approximately $4.2 \times 10^{-6}$ for nanobeads of diameter $100 \mathrm{~nm}$, and no trapping can be achieved when the trapping power is less than $35 \mathrm{~mW}$. The difference of the trapping efficiency between the two types of beads becomes smaller as the size of the particles increase. The trapping efficiency for dielectric beads of diameter $25 \mu \mathrm{m}$ approaches the value predicated by the ray optics model, ${ }^{18}$ while the trapping efficiency for fluorescent beads of the same size is slightly decreased because the focal spot of the laser beam is much smaller than the bead, and only a small fraction of the bead volume is excited. The trapping efficiency of fluorescent beads of diameter $10 \mu \mathrm{m}$ is approximately five times higher than the trapping efficiency of dielectric beads of the same size because the two-photon absorption under the low NA objective occurs within an interaction cross-section of the microbeads larger than the scattering cross-section, due to the greater surface-to-volume ratio. This effect becomes more pronounced when the size of the beads is reduced to $100 \mathrm{~nm}$, in which case the trapping efficiency of the fluorescent nanobeads is approximately 0.01 , which is four orders of magnitude higher than the trapping efficiency of dielectric nanobeads of the same size. The two-photon absorption-enhanced trapping force on the fluorescent nanobeads can facilitate the tweezing of multiple nanobeads within the focal region of a low NA objective, as depicted in Supplementary Fig. S3, which has not been demonstrated by a high NA objective. ${ }^{19-21}$

The discovery of the two-photon absorption-enhanced trapping efficiency of fluorescent nanobeads by low NA ONET is particularly significant when ONET is applied to gold nanorods, which show a strong two-photon absorption cross-section ${ }^{7,22}$ and have been widely used as contrast agents for cancer diagnosis and treatment. ${ }^{7,22,23}$ Thus, we use ONET to trap gold nanorods with an absorption peak at wavelength $800 \mathrm{~nm}$ (see Supplementary Information). Figure 3a1$3 \mathrm{a} 4$ shows that single gold nanorods can be pushed and moved by the trapping beam while they experience Brownian motion (Supplementary Movie 3). The trapping efficiency of a single nanorod is $1.5 \times 10^{-3}$, about three orders of magnitude higher than the trapping efficiency of a single dielectric bead of diameter $100 \mathrm{~nm}$ (Figure 2c). This difference results from the surface plasmon-induced 

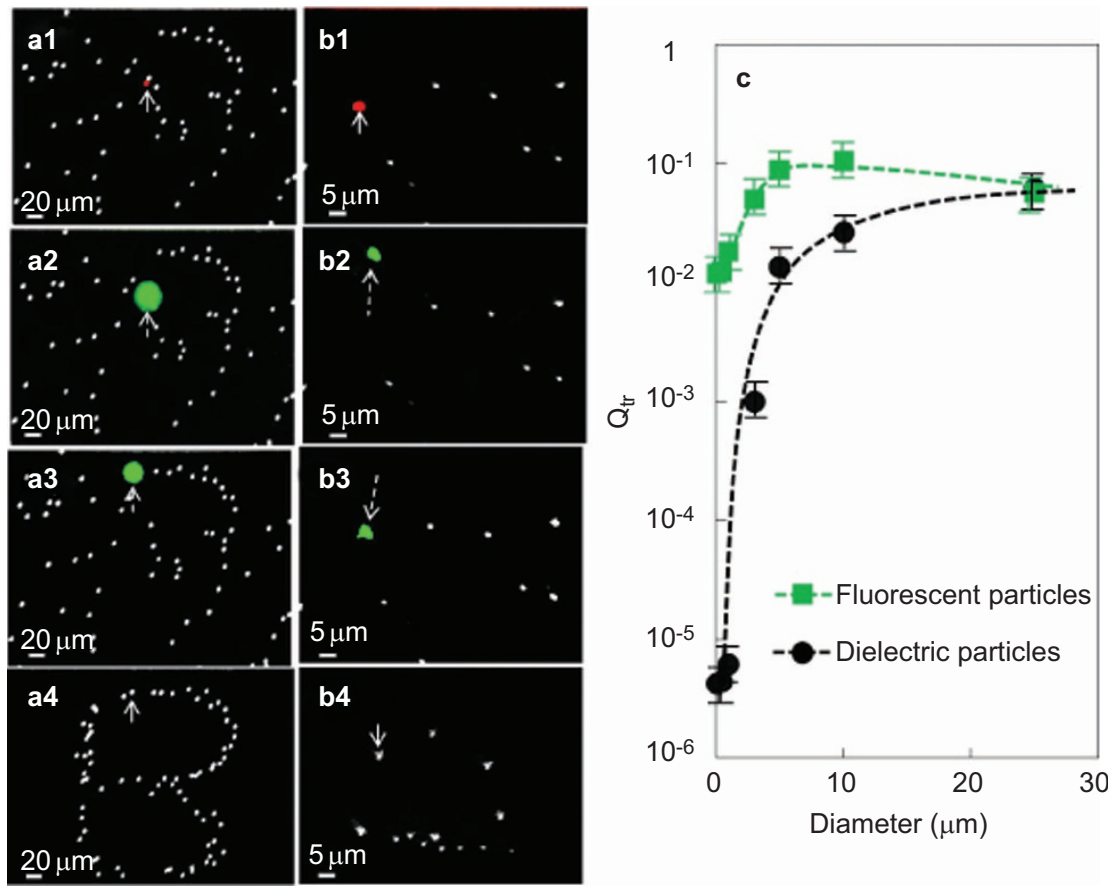

Figure 2 ONETs for fluorescent beads. (a1-a4) Trapped beads of $5 \mu \mathrm{m}$ in diameter were arranged into a letter 'B'. The trapping power is $8.4 \mathrm{~mW}$. (b1-b4) Trapped beads of $100 \mathrm{~nm}$ in diameter were arranged into a semicircle. The trapping power is $8.4 \mathrm{~mW}$. (c) Trapping efficiency of ONET for different diameters of beads. Solid arrows point to the location of the trapping beam, while dashed arrows illustrate the moving paths of the trapping beam. Throughout this paper, the focal spot of the trapping laser beam is colored in red, and the two-photon fluorescence signal is colored in green. ONET, optical nonlinear endoscopic tweezer.

field enhancement and is consistent with the recent theoretical prediction that takes into consideration the photothermal force. ${ }^{24}$ Like absorptive fluorescent nanobeads, nanorods can be pushed toward the first dark ring of the Airy spot. As a result, multiple nanorods can be trapped within the dark ring, as seen in Supplementary Fig. S4, which is similar to the previous observation of trapping gold nanobeads. ${ }^{25-27}$

However, the surface plasmon resonance of gold nanorods within the trapping beam results in a unique feature of ONET as the concentration of gold nanorods increases. Figure $3 \mathrm{~b} 1-3 \mathrm{~b} 4$ and Supplementary Movie 4 show the snowball effect of ONET as the concentration of gold nanorods increases to $10^{3}$ per nanoliter. The following interesting features are observed. First, not only can nanorods near the focal spot be trapped in the focal region but also those from a position up to $4-5$ times the radius of the Airy spot, ${ }^{28}$ which leads to a strong increase in the number of trapped nanorods within the focal region. The trapped gold nanorods can be dragged by the trapping beam. Second, the area in which the gold nanorods are attracted increases with time. The number of trapped nanorods and the critical radius $R$ of the area where nanorods start to move toward the trapping beam are depicted as a function of the trapping time in Figure $3 \mathrm{c}$. Third, for a given time, the moving speed of the gold nanorods within the trapping area is dependent on the radial position (Figure 3d), peaking at the dark ring of the trapping beam and decreasing with distance from the dark ring. For a given radius, the moving speed increases with the trapping time (Figure 3d).

Before we illustrate the physical mechanism for the dynamic snowball effect, it should be pointed out that the distance between the nanorods in Figure 3 is a few micrometers, and thus no coupled plasmonic resonance occurs. ${ }^{29}$ In addition, the power density of the trapping beam in Figure 3 is three orders of magnitude lower than the threshold of generating nonlinear polarization in gold nanoparticles trapped by a high NA objective. ${ }^{30}$ Our experiment also fundamentally differs from the photothermal trapping of dielectric microparticles induced by high-power direct laser heating in water. ${ }^{31}$ Our observations are directly related to the field enhancement caused by the surface plasmon resonance of gold nanorods under two-photon absorption by a low power beam and the subsequent environmental temperature increase caused by heating the nanorods trapped within the focal region. In that sense, our approach is entirely different from the trapping mechanism based on the optical radiation pressure with a negligible temperature gradient force. ${ }^{32}$

Our numerical analysis is based on the generalized FDTD simulation (Supplementary Information). In Figure $3 \mathrm{e}$, we present the electric field around a nanorod illuminated by a focused wave at wavelength $800 \mathrm{~nm}$, showing an enhancement factor up to 3400 . Such a high field enhancement facilitates the nanorod absorption, the heating of the water and the generation of optothermal force beyond the focal region. In the experimental condition for the snowball effect shown in Figure 3, Figure 4a shows that the numerically simulated dependence of the number of gold nanorods trapped in the focus as a function of time is proportional to the cubic power of the trapping time, agreeing well with the experimentally measured dynamic process. The trapped nanorods then absorb a large amount of the energy from the trapping beam, which is converted to heating in the medium (water in the current case) around the focal region and then to temperature increase in the surrounding medium. Figure $4 \mathrm{~b}$ (also see Supplementary Fig. S5) reveals the temperature increase at different locations away from the trapping beam focus as a function of the number of trapped gold nanorods. It is shown that the temperature increase can be above $60 \mathrm{~K}$ with fewer than 300 nanorods trapped in the focus.

Accordingly, the temperature gradient rises (shown in Supplementary Fig. S6), which results in a net optothermal force toward the focus (Figure 4c). It is noted that to attract a nanorod located $5 \mu \mathrm{m}$ away 

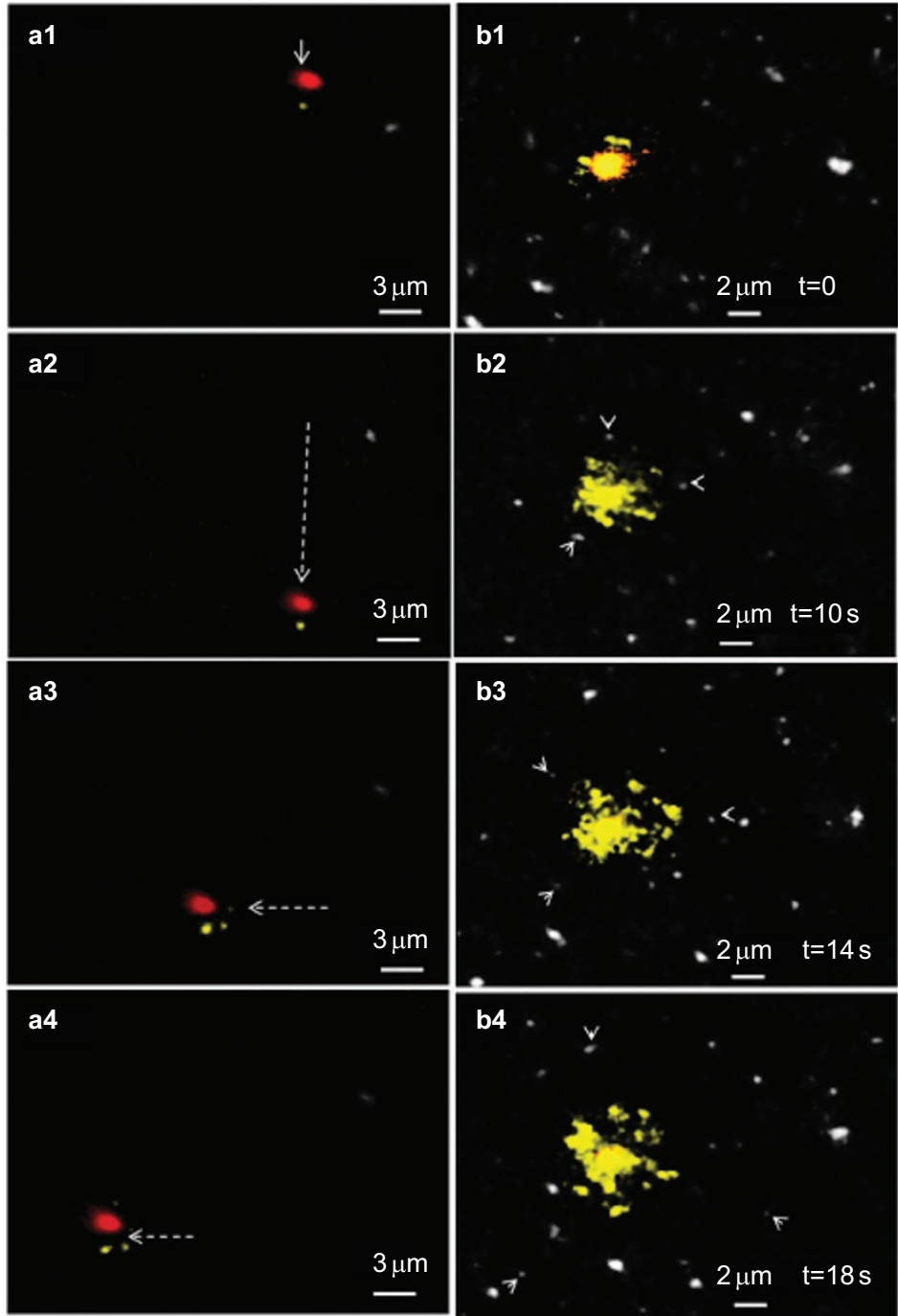

b2
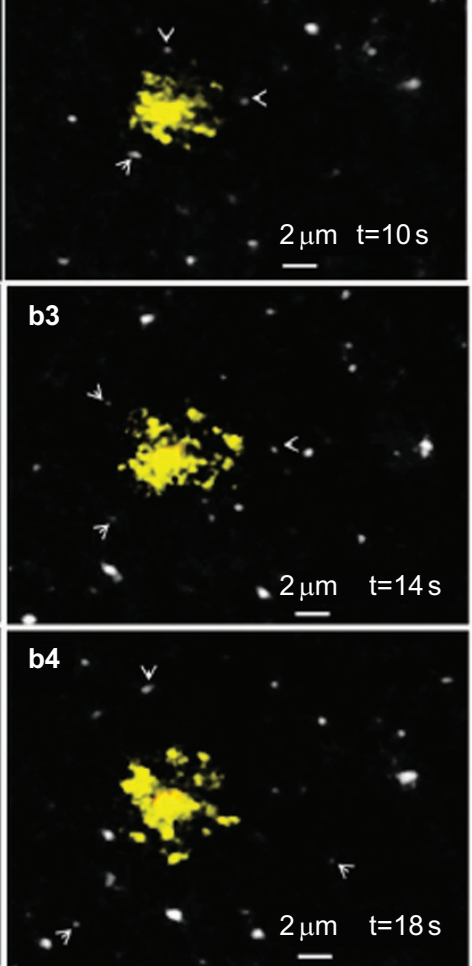
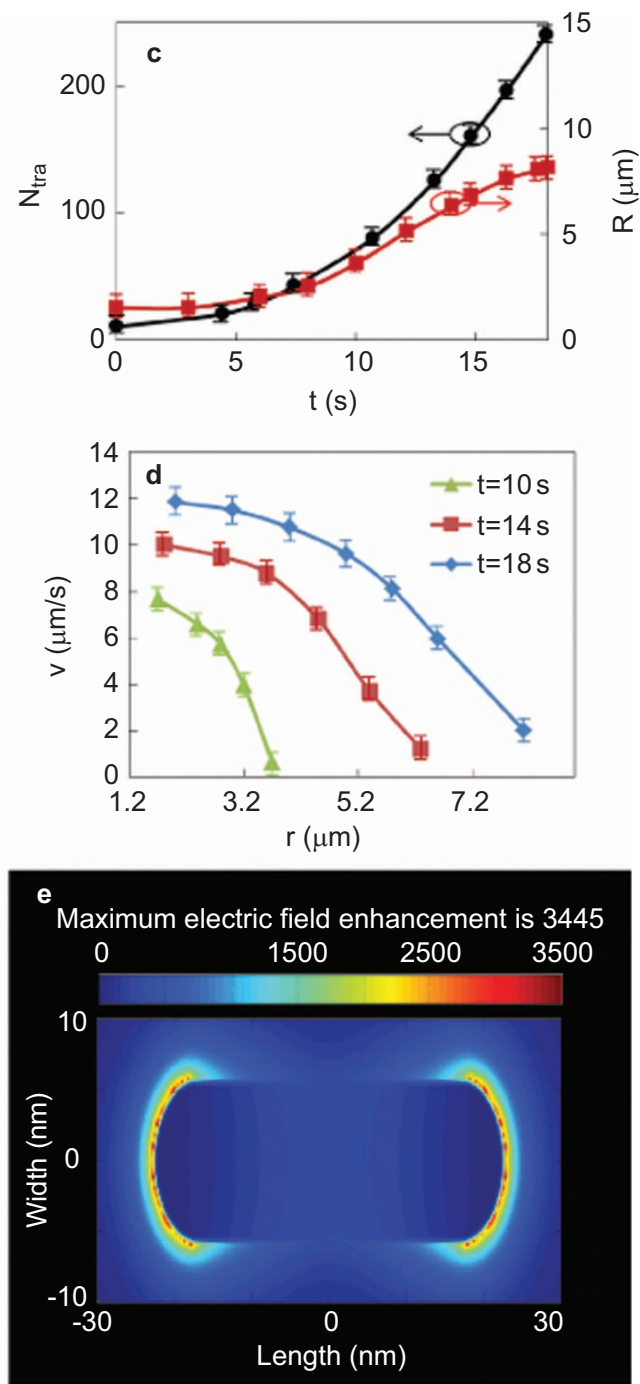

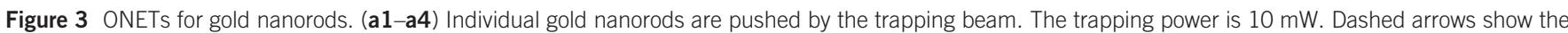

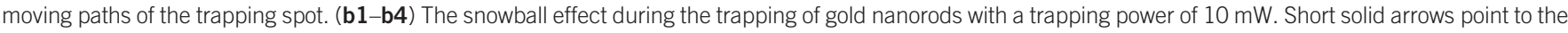

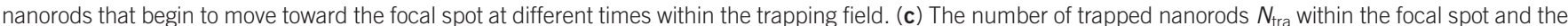

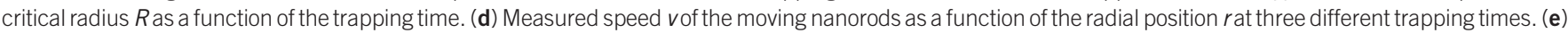
Simulated field enhancement around a nanorod. Throughout this paper, the two-photon-excited photoluminescence from gold nanorods is colored in yellow. ONET, optical nonlinear endoscopic tweezer.

from the focal region, approximately $5 \mathrm{~s}$ are required to build up a pronounced temperature gradient, whereas for a nanorod $10 \mu \mathrm{m}$ away, the required trapping time is $18 \mathrm{~s}$. This prediction quantitatively matches the experimental observations. This optothermal process becomes significantly more pronounced when the number of trapped gold nanorods becomes large (Figure $4 \mathrm{~d}$ ). The optothermal attracting force arising from the temperature gradient can be large enough to overcome the Brownian motion even at a distance 4-5 times the radius of the Airy spot (Figure $4 \mathrm{c}$ and $4 \mathrm{~d}$ ). It is demonstrated that with 250 gold nanorods trapped at the focus, the net photo-thermal force at a place $10 \mu \mathrm{m}$ away from the focus center can reach $0.45 \mathrm{fN}$, which is approximately the threshold to overcome the Brownian motion of the gold nanorods and leads to the observed snowball effect. The trapping of absorptive nanoparticles at the dark ring of the focused laser beam can also be explained by the optothermal effect. When a large number of absorptive nanoparticles have been trapped near the center of the focal region, the heat produced by the absorption can push the temperature near the focal region high enough to produce a repulsive optothermal force, pushing the nanoparticle away from the center of focus. At the same time, the strong electromagnetic field in the focal center pulls the nanoparticles toward the central region. The balanced trapping position at an equilibrium state is very near the dark ring of the Airy spot.

Our numerical prediction that the increase in temperature $\Delta T$ in water around the focal region can be above $60 \mathrm{~K}$ (Figure $4 \mathrm{~b}$ ) implies that the snowball effect generated by the two-photon-induced surface plasmon resonance of the gold nanorods trapped by ONET is potentially promising for individual cell treatment. Although gold nanorods have been widely used for cancer therapy, the specification of gold nanorods for targeting cancer cells is limited by the biofunction technique, requiring more than $6 \mathrm{~h}$ to upload the nanorods to the cancer cells. ${ }^{23}$ Such a long uploading time may induce the risk of complications and increase costs and recovery times for the treatment. Supplementary Fig. S7 demonstrates that ONET could upload the gold nanorods to a targeted cell within 1 min through the snowball 

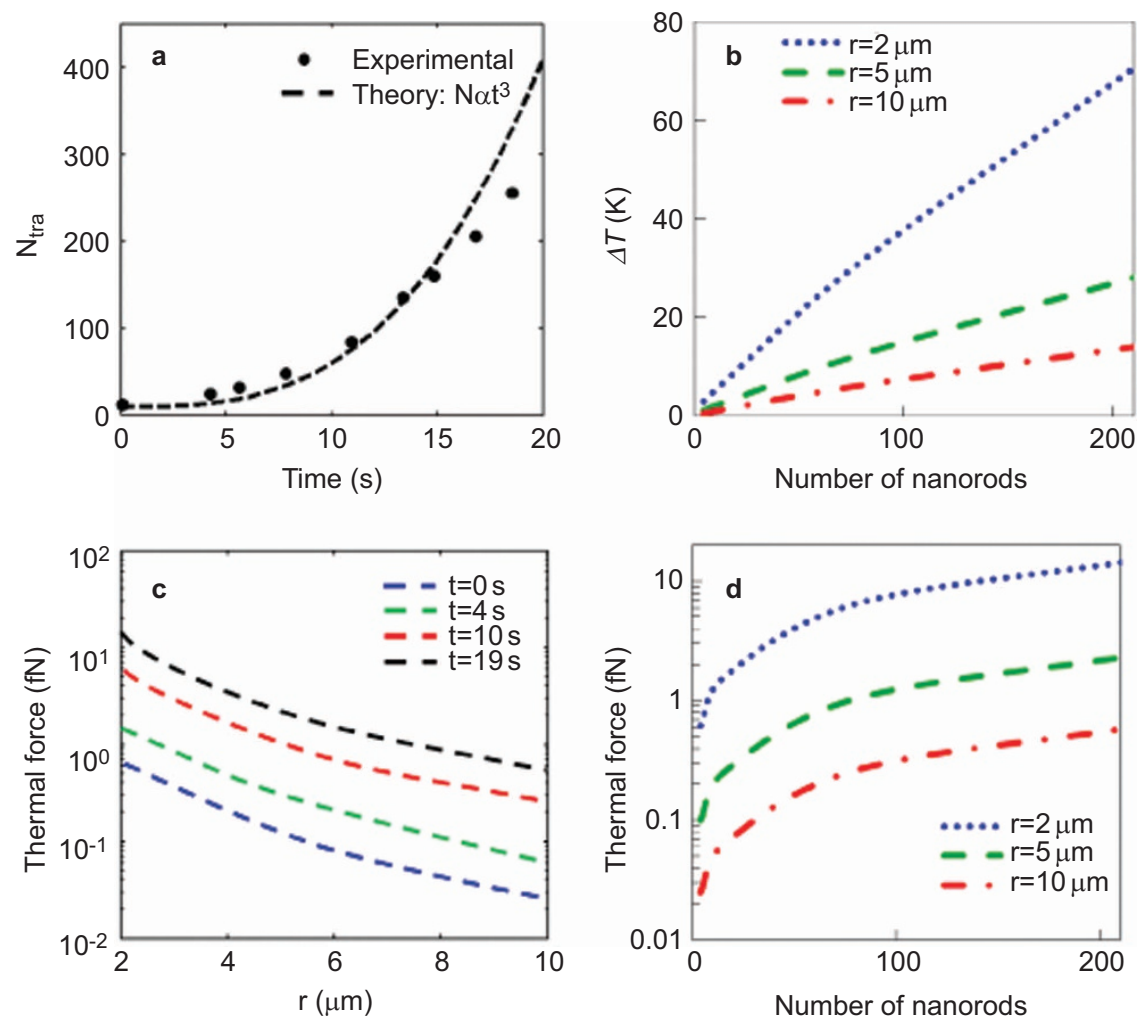

Figure 4 Numerical analysis of the snowball effect. (a) Number of nanorods trapped in the focus as a function of the trapping time. (b) Temperature increase $\Delta T$ as a function of the number of nanorods trapped in the focus. (c) Optothermal force at different trapping times. (d) Optothermal force as a function of the number of nanorods trapped at different radii in the focal plane.

effect. Because our ONET design is based on an optical endoscopy probe connected by a length of fiber, the snowball effect occurring during the course of trapping gold nanorods could be induced within an internal hollow organ for fast individual cell treatment.

\section{CONCLUSIONS}

In this study, we have demonstrated the trapping capabilities of ONET, particularly under two-photon absorption. It has been shown that either aquatic micro- and nanobeads or gold nanorods can be controlled and manipulated efficiently by the ONET device, even though it is equipped with a low-NA probe. Due to their large cross-section, two-photon absorption can enhance the trapping force on fluorescent nanobeads by up to four orders of magnitude compared with the dielectric nanobeads of the same size. The two-photon absorption of the gold nanorods induces an efficient thermal energy conversion, which creates a significant optothermal force to attract nanorods far beyond the laser focal region. This feature allows for the fast loading of a large amount of gold nanorods at a specific target location and the subsequent killing of the cancerous cells. It is important to recognize that the two-photon absorption allows the use of a near-infrared source at wavelength $800 \mathrm{~nm}$, which is at the peak of the transmission window of the tissue environment, effectively reducing the photodamage in the surrounding tissue outside the targeted region. More importantly, the 'snowball effect' enables the dynamic control of the concentration of gold nanorods at the targeted region, greatly reducing the risk factor of loading highly concentrated photosensitizers to achieve a similar photothermal effect. Overall, the ONET device provides a new optical instrument for future in vivo biomedical studies and diagnosis.

\section{AUTHOR CONTRIBUTIONS}

MG proposed the idea of ONET, participated in the experimental design, performed the data analysis and wrote the paper. HB performed the trapping experiment and participated in the data analysis and the paper writing. NS performed the FDTD simulation of two-photon-induced surface plasmon resonance, while XG and JW performed the simulation of the photothermal attracting force. All authors contributed to the paper writing through discussion.

\section{ACKNOWLEDGMENTS}

The authors thank Jingliang Li for his help in the synthesis of gold nanorods. We appreciate the discussion with Fuxi Gan from the Shanghai Institute of Optics and Fine Mechanics.

1 Ashkin A. Acceleration and trapping of particles by radiation pressure. Phys Rev Lett 1970; 24: 156-159.

2 Ashkin A, Dziedzic JM. Optical trapping and manipulation of viruses and bacteria. Science 1987; 235: 1517-1520.

3 MacDonald MP, Spalding GC, Dholakia K. Microfluidic sorting in an optical lattice. Nature 2003; 426: 421-424.

4 Hubbell JA. Enhancing drug function. Science 2003; 300: 595-596.

5 Huang XH, El-Sayed IH, Qian W, El-Sayed MA. Cancer cell imaging and photothermal therapy in the near-infrared region by using gold nanorods. J Am Chem Soc 2006; 128: 2115-2120.

$6 \mathrm{Gu}$ M, Bao H, Li JL. Cancer-cell microsurgery using nonlinear optical endomicroscopy. J Biomed Opt 2010; 15: 050502.

7 Durr NJ, Larson T, Smith DK, Korgel BA, Sokolov K et al. Two-photon luminescence imaging of cancer cells using molecularly targeted gold nanorods. Nano Lett 2007; 7: 941-945.

8 Lyubin EV, Khokhlova MD, Skryabina MN, Fedyanin AA. Cellular viscoelasticity probed by active rheology in optical tweezers. J Biomed Opt 2012; 17: 101510.

9 Chung HS, McHale K, Louis JM, Eaton WA. Single-molecule fluorescence experiments determine protein folding transition path time. Science 2012; 335: 981-984. 
10 Ashkin A. Applications of laser radiation pressure. Science 1980; 210: 10811088.

11 Moffitt JR, Chemla YR, Izhaky D, Bustamante C. Differential detection of dual traps improves the spatial resolution of optical tweezers. Proc Natl Acad Sci USA 2006; 103: 9006-9011.

12 Zhang W, Huang L, Santschi C, Martin OJ. Trapping and sensing $10 \mathrm{~nm}$ metal nanoparticles using plasmonic dipole antennas. Nano Lett 2010; 10: 10061011 .

13 Neuman KC, Block SM. Optical trapping. Rev Sci Instrum 2004; 75: 2787-2809.

14 Flusberg BA, Cocker ED, Piyawattanametha W, Jung JC, Cheung EL et al. Fiber-optic fluorescence imaging. Nat Methods 2005; 2: 941-950.

15 Bao H, Allen J, Pattie R, Vance R, Gu M. A fast handhold two-photon fluorescence micro-endoscope with a $475 \mu \mathrm{m} \times 475 \mu \mathrm{m}$ field of view for in vivo imaging. Opt Lett 2008; 33: 1333-1335.

16 Cizmar T, Dholakia K. Shaping the light transmission through a multimode optical fibre: complex transformation analysis and applications. Opt Express 2011; 19: 18871-18884.

17 Konig K, Ethlers A, Riemann I, SchenkI S, Buckle R et al. Clinical two-photon microendoscopy. Micros Res Tech 2007; 70: 398-402.

18 Ganic D, Gan X, Gu M. Optical trapping force with annular and doughnut laser beams based on vectorial diffraction. Opt Express 2005; 13: 1260-1265.

19 Liu Y, Sonek YG, Berns MW, Konig K, Tromberg B. Two-photon fluorescence excitation in continuous wave infrared optical tweezers. Opt Lett 1995; 20: 2246-2248.

20 Agate B, Brown CT, Sibbett W, Dholakia K. Femtosecond optical tweezers for in-situ control of two-photon fluorescence. Opt Express 2004; 12: 3011-3017.

21 Morrish D, Gan X, Gu M. Morphology-dependent resonance induced by two-photon excitation in a micro-sphere trapped by a femtosecond pulsed laser. Opt Express 2004; 12: 4198-4202.
22 Wang HF, Huff TB, Zweifel DA, He W, Low PS et al. In vitro and in vivo two-photon luminescence imaging of single gold nanorods. Proc Natl Acad Sci USA 2005; 102 : $15752-15756$.

23 Li JL, Day D, Gu M. Ultra-low energy threshold for cancer photothermal therapy using transferrin-conjugated cold nanorods. Adv Mater 2008; 20: 3866-3871.

24 Wu J, Gan X. Optimization of plasmonic nanostructure for nanoparticle trapping. Opt Express 2012; 20: 14879-14890.

25 Dienerowitz M, Mazilu M, Reece PJ, Krauss TF, Dholakia K. Optical vortex trap for resonant confinement of metal nanoparticles. Opt Express 2008; 16: 4991-4999.

26 Furukawa H, Yamaguchi I. Optical trapping of metallic particles by a fixed Gaussian beam. Opt Lett 1998; 23: 216-218.

27 Gu M, Ke PC. Image enhancement in near-field scanning optical microscopy with laser-trapped metallic particles. Opt Lett 1999; 24: 74-76

28 Gu M. Advanced Optical Imaging Theory. Heidelberg: Springer; 2000.

29 Zelenina A, Quidant R, Nieto-Vesperinas M. Enhanced optical forces between coupled resonant metal nanoparticles. Opt Lett 2007; 32: 1156-1158.

30 Jiang Y, Narushima T, Okamoto $\mathrm{H}$. Nonlinear optical effects in trapping nanoparticles with femtosecond pulses. Nat Phys 2010; 6: 1005-1009.

31 Xin H, Li X, Li B. Massive photothermal trapping and migration of particles by a tapered optical fiber. Opt Express 2011; 19: 17065-17074.

32 Deng H, Li G, Dai Q, Ouyang M, Lan S et al. Role of interfering optical fields in the trapping and melting of gold nanorods and related clusters. Opt Express 2012; 20: 10963-10970.

(c) (i) (2) This work is licensed under a Creative Commons AttributionNonCommercial-ShareAlike 3.0 Unported license. To view a copy of this license, visit http://creativecommons.org/licenses/by-nc-sa/3.0

Supplementary Information for this article can be found on Light: Science \& Applications' website (http://www.nature.com/lsa/). 